\title{
Economía y Mujer: vínculos existentes
}

- Lilian Vega

Lo que trataré de hacer es exponer algunos puntos de reflexión sobre los diferentes vínculos que pueden existir entre la economía y temas relacionados con el bienestar de la mujer; dado que son reflexiones, las ideas que expondré las haré a título personal. La exposición, trataré de hacerla abordando primero aspectos relacionados con los diferentes vínculos o relaciones que pueden existir entre temas relacionados clásicamente con aspectos económicos y sus efectos sobre el bienestar de las mujeres. En un segundo momento, expondré algunos aspectos que mediatizan la forma como se abordan académicamente a través de investigaciones, metodologías o estadísticas estos vínculos para luego poder traducirlas en acciones concretas que beneficien a las mujeres.

La primera reflexión gira en torno a que los mercados, sean financieros, de bienes públicos, bienes transables, servicios o cualquiera del que se trate, no interiorizan dentro de su funcionamiento para lograr la eficiencia - suponiendo que en realidad ésta se logra - cómo afecta el bienestar de la mujeres; tampoco la mayoria de los y las economistas tomamos como punto de partida de análisis o de consideración el interés de la situación de las mujeres. Sin embargo, se debe reconocer que las cosas han mejorado un tanto a lo largo del tiempo: por ejemplo, existen movimientos civiles y voluntad para lograr un mejor entendimiento entre economistas y grupos que trabajan por mejorar las condiciones de vida de las mujeres y, para visibilizar y concientizar a la sociedad que la equidad en términos de género es beneficiosa, no sólo desde el punto de vista ético y humanista —en tanto hombres y mujeres somos seres humanos de igual calidadsino también, desde el punto de vista puramente económico. Conceptos básicos como la retribución sobre la base de la productividad marginal sin distinción de género, apuntan a la equidad sin distinción o discriminación debido al sexo de la 
persona. Aunque estamos todavía lejos de alcanzarla, el camino ya se ha empezado a recorrer.

Una de las tareas en las que falta trabajar o profundizar es hacer entender que no existe un conflicto entre la economía y la visión de género, sino al contrario: que el sesgo negativo o de discriminación en aspectos económicos, es nocivo y perjudica a la economía, sobre todo si a lo que aspiramos es lograr un crecimiento económico que potencie el desarrollo humano.

La intención es mostrar algunos vínculos existentes entre diferentes aspectos de la economía y el bienestar de las mujeres. Posiblemente muchas de las ideas que voy a exponer aquí resulten evidentes. Sin embargo, a veces es necesario remarcar aquellos aspectos que por su misma evidencia e interiorización inconsciente pasan desapercibidos para la mayoria de nosotros, y en ese sentido poco o nada se avanza en el caminar que hemos iniciado.

\section{Políticas macroeconómicas: Influencia en el bienestar de las mujeres}

Es válido preguntarse acera del vínculo que existe entre economía y mujer, más allá de presentar estadísticas clasificadas según sexo. En esta línea de pensamiento es posible postular que las políticas económicas no son neutrales, pues tienen impactos diferenciados en las personas, dependiendo de características como edad, profesión o sexo. Aun aquellas políticas que aparentemente no discriminan o cuyos efectos se perciben como mínimos pueden tener impactos importantes sobre los intereses o bienestar de las mujeres. Las politicas inflacionistas pueden tener un impacto menos negativo sobre el bienestar de las mujeres, que la disminución de la cantidad del presupuesto asignada y efectivamente ejecutada en programas como el ISDEMU o en campañas de salud por ejemplo, sobre todo si se trata de mujeres que están endeudadas - posiblemente por un objetivo de poner una microempresa- porque les permite disminuir su deuda en términos reales, es decir, dado que en términos reales o de poder adquisitivo de la moneda posiblemente se encuentre pagando menos, mientras que la capacidad de cobertura que puedan tener las acciones del ISDEMU no necesariamente son de amplia base. Por otro lado, políticas antiinflacionarias implican normalmente a aumentos de las tasas de interés, al suceder esto, se tiende a perjudicar a las mujeres jóvenes que buscan o tienen empleo en las maquilas por ejemplo, debido a los efectos de este tipo de políticas sobre el empleo (desempleo) y la producción, además de que resulta también que las personas que tienen deudas están pagando más en intereses. Claro, la cuantificación de éstos impactos es un tema que sobrepasa los objetivos de esta reflexión; otras relaciones similares pueden establecerse con las políticas comerciales y cambiarias.

El punto sobre el cual se quiere llamar la atención es que las políticas económicas pueden afectar de diversas maneras a las mujeres y su situación, existen 
diferentes niveles que van desde efectos directos, o sea políticas elaboradas de manera explícita para mejorar las condiciones de las mujeres o algún grupo de mujeres, hasta las políticas con efectos indirectos, como las políticas monetaria, fiscales, cambiarias, comerciales o el mismo proceso de globalización.

Normalmente son fuente de discusión o debate aquellas políticas, programas o estrategias cuya influencia es directa en las mujeres o sobre algún grupo de mujeres hacia quiénes se han focalizado los esfuerzos, por ejemplo, políticas de apoyo directo en financiamiento a micro y pequeñas empresas dirigidas por mujeres o campañas de salud'. Sin embargo, sería conveniente y altamente deseable dirigir la atención a los impactos que las políticas más generales puedan tener sobre el bienestar de las mujeres (y otros grupos vulnerables o importantes como los niños, en quienes descansa el futuro de nuestro país). Así, la política para promover la inversión foránea, que, tal como ha respondido la empresa privada extranjera, es un claro ejemplo de un resultado de una política aparentemente neutral, pero cuyo efecto ha sido mucho mayor en las mujeres que en los hombres, dado que la respuesta principal a esta politica ha sido el establecimiento de maquilas textiles, que emplean mayoritariamente mano de obra femenina, lo que ha conducido por ejemplo a que se incorpore al mercado laboral a una gran cantidad de mujeres que antes no lo consideraban como opción ${ }^{2}$-más allá de que ésta sea la mejor opción posible - y a que, en general, los niveles de desempleo femenino sean menores que los masculinos.

\section{Ajuste estructural y bienestar de las mujeres}

Mucho se ha hablado y escrito sobre las políticas y resultados que ha tenido el programa de ajuste estructural que se llevó a cabo a inicios de la década de los noventa. Sin embargo, existen varios vínculos poco abordados sobre el desarrollo e impacto de este proceso. Por ejemplo: el existente entre ajuste estructural y bienestar de las mujeres. De manera general, se puede indicar que realizar ajustes estructurales significa cambiar los precios relativos entre trabajo remunerado y no remunerado a favor del primero debido a la preponderancia que adquiere el mercado. De esta manera, se contribuye a invisibilizar el trabajo realizado por las mujeres en las tareas reproductivas y de cuidado del hogar e hijos, desvalorizándolas aún más, comparadas con la producción del mercado. Resultado: los términos de intercambio intradomésticos de comercio se deterioran para las mujeres debido a la mayor preponderancia de las actividades remuneradas, o "productivas", desde el punto de vista del mercado.

Aclarando un poco más cómo ha sucedido lo anterior podemos indicar por ejemplo que parte de los programas de ajuste estructural incluyen políticas como la introducción de pagos para los usuarios de la salud primaria y educación brindada por el Estado; esta medida implica un incremento de la presión sobre los ingresos, sobre todo si se trata de unidades familiares pobres, por consi- 
guiente, también aumenta la necesidad de encontrar un trabajo remunerado aunque sea en el sector informal y sin las prestaciones sociales a las que se tiene acceso en el sector formal típicamente salud y previsión social. Muchas mujeres deciden entrar al mercado laboral con una combinación perversa de subempleo y sobreexplotación, dado que pueden llegar trabajar más de ocho horas diarias y percibir ingresos menores a los salarios mínimo, lo que en las estadisticas de desempleo se conoce con el nombre de "desempleo invisible". Obviamente, esto empeora la situación de bienestar de muchas mujeres en los estratos pobres de la población, al exigirles una mayor esfuerzo en trabajo "remunerado" de baja calidad e ingreso, sin que ello signifique una disminución del trabajo reproductivo; la alternativa es disminuir aún más o incluso perder acceso a bienes y servicios básicos, como la salud preventiva, por ejemplo; este fenómeno es más sentido por una gran cantidad de mujeres que son jefas de hogar y que, por consiguiente, son responsables de obtener ingresos para ellas y sus familias.

El fenómeno anterior no sólo afecta a las mujeres, sino también (y fuertemente) a los niños y niñas que dependen de ellas, pues, al salir la madre a trabajar pierden mucho del tiempo que ella podria dedicarles en su cuidado en general, parecería que existen condiciones para empeorar la situación de estas familias.

Las mujeres enfrentan precios relativos muy desfavorables para ellas, pues, por un lado, el trabajo realizado en el hogar no es valorizado por ningún mercado, dado que no puede ser incorporado a ninguna cadena de producción de algún bien o servicio, aún cuando existen estudios que tratan de cuantificarlo y demuestran que significan un alto porcentaje de Producto Interno producido en un pais. Por otro lado, las politicas de ajuste estructural han llevado a configurar una economía en la que se marca una tendencia al crecimiento del sector informal y a una creación de empresas que demandan mano de obra con poca calificación y cierta habilidad manual. Ambas tendencias crean puestos de trabajo aptos para mujeres, lo que un principio apuntaria en general a mejorar el bienestar de las mismas al permitirles acceso a una actividad remunerada. El problema es que la calidad del puesto de trabajo creado no es lo suficientemente bueno como para que el salario y/o las condiciones, laborales enfrentadas permitan un desarrollo laboral beneficio; más bien, las tendencias parecerian que van en el sentido de generar puestos de trabajo en el sector formal con remuneraciones bajas, en el mejor de los casos o, puestos de trabajo en el sector informal en dónde además de la baja remuneración presentan características de subempleo y ausencia de acceso a las prestaciones de salud y previsión social. Es así como muchas mujeres entran en el mercado laboral, en las condiciones apuntadas, sin que ello signifique poder contar con una disminución efectiva del trabajo reproductivo dentro del hogar. Es totalmente previsible que la calidad del tiempo, y el trabajo dedicado a la formación de los hijos e hijas y, en general, al mantenimiento de un ambiente familiar óptimo para el desarrollo humano sufre 
una disminución que afecta a las mujeres, a todo su grupo familiar y al pais en general.

\section{Crecimiento económico y mujeres: Relación biunívoca}

Hasta aquí hemos hecho referencia los efectos que algunas políticas y programas económicos pueden tener sobre las mujeres. Falta reflexionar sobre la causalidad inversa; es decir, cómo el bienestar de las mujeres afecta el crecimiento económico en calidad y cantidad, o mejor dicho, cómo afecta el desarrollo humano que se puede lograr en un pais. Existen vínculos que sí han sido objeto de tratamientos más extensos, por ejemplo, la relación positiva que existe entre crecimiento económico y bienestar de la población en general y de las mujeres en particular.

Los informes de desarrollo humano por ejemplo, tratan de evidenciar estas relaciones e incluso van más allá, obteniendo Índices de Desarrollo Humano relativo al Género (IDG) y el Índice de Potenciación de Género (IPG). Este último, capta sobre todo la desigualdad de género en tres áreas claves de representación y de toma de decisiones políticas y económicas: i) la participación política y en la toma de decisiones, ii) la participación económica y poder, y iii) en la toma de decisiones y control sobre los recursos económicos. Para lo anterior, utilizan variables como la representación de las mujeres en los consejos municipales, puestos administrativos y ejecutivos, puestos profesionales y técnicos, y PIB per cápita de hombres y mujeres. Estos son esfuerzos sistemáticos encaminados a evidenciar la situación de bienestar de las mujeres.

Pero, volviendo a la relación con el crecimiento económico, la causalidad se da en ambas direcciones: el crecimiento económico es favorable para el desarrollo y bienestar de las mujeres, a la vez más las mujeres con mayores calidades de vida potencian el crecimiento económico al volverse más productivas, más eficientes en la utilización del tiempo que dedican y a su vez en potenciar mejor el desarrollo de sus propias capacidades y de sus hijos.

Se ha demostrado que una alta tasa de crecimiento, sobre todo, si va acompañada de igualdad de ingresos y oportunidades sin discriminaciones de ningún tipo, incluyendo género, es importante para lograr altas tasas de crecimiento; existe una correlación positiva bastante alta entre un mayor nivel de equidad y mayores tasas de crecimiento. Lograr una tasa de crecimiento sostenible en el tiempo necesita de un nivel de equidad aceptable, al mismo tiempo que la existencia de desigualdad de ingresos debido a género es un factor importante en los niveles de pobreza y bienestar de las mujeres.

Con lo anterior, se quiere indicar que el crecimiento no es suficiente para mejorar el bienestar de las mujeres. Si bien, el mero crecimiento es favorable 
para este objetivo, se necesita de mayor equidad en la distribución del ingreso, de poder y control sobre los recursos económicos entre hombres y mujeres. Como lo indica Stefan de Vylder, "una distribución desigual de los ingresos refleja una distribución desigual de poder", es necesario avanzar en un enfoque democrático y participativo que permita el empoderamiento de las mujeres y redunde en un mayor bienestar para todas nosotras. Nuevamente, los aspectos cualitativos del crecimiento, relacionados con el concepto de desarrollo humano son tanto o más importantes que el crecimiento en sí mismo para mejorar la calidad de vida de la mujeres.

\section{Superar la barrera de los datos}

Como hemos visto, existen una variedad de vínculos entre economía y mujer que pueden ser desarrollados conceptualmente de una manera aceptable, es necesario luego trascender la elaboración conceptual y pasar a evidenciar empíricamente estas relaciones. Aquí entramos al terreno de las dificultades con que se topan los investigadores para demostrar o concretizar los desarrollos conceptuales.

La primera dificultad está relacionada con la falta o carencia de estadísticas fidedignas que permitan realizar un análisis profundo del presupuesto del gobierno y su influencia sobre el bienestar de las mujeres. Esta dificultad procede de la forma en la cual se clasifica, ordena o presenta la información, la cual en este ejemplo en particular, está enfocada a permitir análisis de la estructura económica, de las macroáreas de gestión o de los órganos del Estado, es decir no es adecuada para analizar, diseñar y poner en práctica políticas apropiadas en función de las mujeres. La forma de presentación del presupuesto es sólo un ejemplo, pero en realidad no existe una sistematización institucionalizada de estadisticas desglosadas por género, situación económica y otras variables importantes para darle seguimiento a los temas relacionados con el bienestar de la mujeres, incluso las estadísticas sociales y económicas básicas no siempre están desglosadas por género y los datos sobre la salud de las poblaciones a menudo reducen el tema de salud de la mujer en lo concerniente a la reproducción.

Otro ejemplo de problemas con el manejo de información es lo concerniente a estadísticas laborales, la invisibilización del trabajo femenino es problemática y algunas de las causas son estructurales, por ejemplo mucha de la población femenina empleada lo hace en el sector paralelo o informal de la economía, sector en el cual la generación de estadísticas es claramente deficiente, además, casi ninguna fuente de información primaria permite obtener datos sobre el trabajo reproductivo que realizan las mujeres y lo que eso significa en términos económicos.

La información y las estadísticas fidedignas sobre género son indispensables para el estudio de la manera en que los factores económicos (también sociales y 
del medio ambiente) afectan las tendencias en el desarrollo de las mujeres o cómo son afectados por éstas. La recopilación y el análisis apropiados de los datos sobre esas complejas interacciones tienen importancia crítica para que los encargados de formular políticas puedan preparar programas que respondan a las necesidades de quienes han de ser beneficiarias de dichos programas.

Se han realizado algunos esfuerzos por parte de instituciones internacionales como los programas de Naciones Unidas para subsanar estos problemas, creando metodologias como la propuesta por CEPAL en 1999 para el seguimiento de la IV Conferencia de la Mujer en el Área Mujer y Economía. En este caso, se crearon metodologías e índices relacionados con los objetivos estratégicos planteados en la conferencia de Beijing. Todos apuntan a la equidad de género en varios ámbitos como el mercado laboral, el sector informal de la economía y pequeñas empresas, acceso a ocupaciones especializadas y puestos de dirección y participación en las actividades reproductivas realizadas en el ámbito doméstico. En esta misma línea de esfuerzo también podemos mencionar los índices de Potenciación de Género y de Desarrollo Humano relativo al Genero elaborados en los Informes de Desarrollo Humano.

Pero la recopilación y creación de datos que sean comparables a escala internacional es sólo el comienzo del proceso, claramente necesario pero insuficiente. Es necesario que los datos estén adecuadamente analizados, ampliamente difundidos y oficialmente interesados en la planificación del desarrollo; y todo ello requiere conocimientos complejos y especializados $y$, por consiguiente, capacitación técnica.

Sin duda, la universidad, en tanto espacio destinado a la formación de los futuros profesionales y centro creador y transmisor de conocimientos, es un ámbito fundamental para los nuevos temas y teorías. El reconocimiento académico de ellos es una forma de validación social que complementa, y de una u otra manera legitima, lo avanzado. Esto tiene una gran relevancia pues algunos esfuerzos encaminados a producir conocimiento sobre el tema, han sido cuestionados y se ha intentado marginarlos por considerárselos muy ideologizados, o con relaciones demasiado estrechas con el feminismo. Es así entonces como la instalación de los Estudios de la Mujer y el Género en las universidades es una condición indispensable para formar a los investigadores que más adelante abordarán estos temas y para sensibilizar a los futuros cuadros profesionales que en el día de mañana desde el Estado, los gobiernos locales o la sociedad civil estarán diseñando e implementando las políticas públicas. De tal modo que su puesta en marcha no sólo tiene resonancias en lo académico, sino que establece una relación dialéctica con lo político.

Como hemos dicho, han existido avances y es posible mirar hacia atrás y ver cuánto se ha logrado. Actualmente existe información de diferente tipo que per- 
mite hacer balances y medir los avances y posicionamiento de los temas de la mujer y economía Hay investigaciones que dan cuenta de la participación politica y económica de las mujeres. Sin embargo, aún hay ámbitos en los cuales persiste la desinformación y uno de ellos es el universitario. Esto no es dificil de explicar si se considera que estos conocimientos surgieron y se desarrollaron bastante al margen de esos espacios. Desde mi perspectiva muy personal, es importante avanzar en este esfuerzo en las aulas, en las tesis que se elaboran y en la investigación académica que se realice.

Los Estudios de la Mujer permiten visibilizar a un amplio sector social, dando a conocer la particular condición en que se desenvuelven sus vidas en diversas esferas y medios sociales. Las mujeres y su relación con el trabajo, con el desarrollo, con la educación, la salud mental y la salud del cuerpo son algunos de los aportes de estos estudios al conocimiento. Tal vez uno de los mayores desafios que enfrentan los estudios de género es contribuir a la realización de análisis más integrales que permitan aprehender de manera más fina los complejos procesos y establecer cómo los sujetos los viven y los representan. Creo posible y necesario hoy que los estudios de la mujer y la economía, profundicen una discusión sobre aspectos teóricos y metodológicos que permita ir generando un modo de pensar cientifico que nos permitan conocer dónde y cómo se reproducen las desigualdades entre hombres y mujeres, para poder actuar sobre ellas en la búsqueda de una equidad que nos beneficiará a todos.

\section{Notas}

1. En este punto considero pertinente indicar que las campañas de salud y la salud en general de la mujeres es concebida normalmente por las autoridades circunscrita a la salud reproductiva, lo que evidencia la falta de una visión integral hacia las mujeres.

2. La tasa global de participación femenina ha aumentado de $33.80 \%$ en 1992 a $39.12 \%$ en 1999, la cual corresponde al porcentaje de mujeres en edad de trabajar que se encuentran económicamente activas, esto es, empleadas, o buscando activamente un empleo. 